\title{
ARTE RUPESTRE EN LA CUENCA DEL RÍO MOJONES (ANTOFAGASTA DE LA SIERRA, CATAMARCA, ARGENTINA)
}

\section{ROCK ART IN MOJONES RIVER BASIN (ANTOFAGASTA DE LA SIERRA, CATAMARCA, ARGENTINA).}

\author{
María Cecilia Gentile${ }^{1}$, Martín Tomás Casanova Menéndez², Jennifer Grant ${ }^{3}$ Luis Coll ${ }^{4}$ y \\ Kevin Lane ${ }^{5}$ \\ ${ }^{1}$ INAPL - CONICET, 3 de febrero 1370, C.A.B.A. E-mail: chi.gentile@hotmail.com \\ ${ }^{2}$ INAPL - CONICET, 3 de febrero 1370, C.A.B.A. E-mail: martincas89@hotmail.com \\ 3INAPL - CONICET, 3 de febrero 1370, C.A.B.A. E-mail: jennygrantlett@gmail.com \\ ${ }^{4}$ Museo Etnográfico "Juan B. Ambrosetti" - UBA, Moreno 350, C.A.B.A. \\ E-mail: luisvjcoll@hotmail.com \\ 5Instituto de Arqueología - CONICET, 25 de mayo 221, C.A.B.A. E-mail: kevin.lane@cantab.net
}

$$
\text { Presentado: 06/06/2019_Aceptado: 07/08/2019 }
$$

\section{Resumen}

En este trabajo se exponen los resultados preliminares obtenidos del análisis del arte rupestre del sitio Corral Grande 1, ubicado en Antofagasta de la Sierra, un oasis de altura situado en la Puna meridional Argentina. Se presenta una descripción de las representaciones analizadas y se les da un marco contextual al asociarlas con otros casos similares de la región de estudio. Los resultados del análisis son discutidos en relación con los modelos de asentamiento y subsistencia formulados para el Período Formativo en la microrregión (ca. 3,000-1,100 años AP). Teniendo en cuenta dichos modelos, proponemos una posible interpretación de los motivos en asociación con las actividades que conformaron la base económica de las sociedades andinas tempranas: el pastoreo de camélidos y la producción agrícola.

Palabras Clave: Puna de Atacama; Arte Rupestre; Período Formativo; Sociedades Agroalfareras; Representación del agua.

\section{Abstract}

This paper presents preliminary results on rock art from the site Corral Grande 1, located in Antofagasta de la Sierra, Catamarca, Argentina. We describe the analysed motifs and provide a regional framework based on a comparison with similar cases within the study region. The results obtained from this analysis are then discussed in relation to the settlement and subsistence models proposed for the early societies that populated the area (ca. 3,000-1,100 years BP). Bearing in mind 
these models, we propose an interpretation for some of the motifs in relation to the main economic activities of early Andean societies: camelid pastoralism and agricultural production.

Key Words: Puna of Atacama; Rock art; Formative period; Agricultural-pottery societies; Representation of water.

\section{Introducción}

En la microrregión de Antofagasta de la Sierra (en adelante ANS) se han llevado a cabo investigaciones durante más de dos décadas. A un nivel general, el objetivo de estos trabajos fue comprender los diferentes procesos sociales y económicos atravesados por las poblaciones que allí llevaron a cabo sus actividades. De esta forma, se pudo establecer que las primeras poblaciones de las que se tiene registro fueron grupos cazadores-recolectores de una alta movilidad logística y residencial. Hacia ca. 4500 años AP se inició un proceso de sedentarización e incorporación de la opción productiva (Olivera 2012), con los consecuentes cambios socioculturales que dicha transición puede traer aparejados. De esta manera, entre ca. 3000-1100 años AP la evidencia presenta sociedades formativas ya consolidadas.

Los primeros trabajos respecto de estos grupos están centrados en el sitio Casa Chávez Montículos, el cual fue interpretado como una base residencial de actividades múltiples (Olivera 1992). Posteriores investigaciones realizadas en otros sectores dentro de la microrregión (Escola et al. 2015) permitieron detectar nuevas ocupaciones asignables a este momento, lo cual generó un visión más compleja y acabada de la que se tenía previamente respecto del Formativo local. Asimismo, trabajos de campo llevados a cabo en los años 2010 y 2018 permitieron identificar nuevas áreas con evidencias arqueológicas pertenecientes al Período Formativo. En uno de estos sectores se ubica el complejo de sitios Corral Grande, localizado en la cuenca del río Mojones, hacia el oeste de la microrregión (Figura 1). El mismo está compuesto por una serie de sitios arqueológicos que cuentan con evidencia arquitectónica, cerámica, lítica, ósea y de arte rupestre que permiten asignar las ocupaciones desde momentos formativos hasta entrado el Período Colonial.

El presente trabajo se inserta dentro de los estudios del Período Formativo en ANS con el objetivo de dar a conocer el arte rupestre identificado en el sitio Corral Grande 1 durante las prospecciones realizadas en el año 2010. Como objetivo complementario, buscamos dar un paso en la interpretación del posible significado de algunos de los motivos observados en los paneles del sitio. Para ello, consideramos sus características morfológicas y estilísticas, su relación con el arte rupestre a nivel regional, su disposición espacial y su visibilidad en relación con la arquitectura del sitio del cual forman parte. Además, se tomaron en cuenta información contextual del sitio obtenida de las excavaciones, ejemplos etnográficos y trabajos arqueológicos centrados en los rituales de producción agrícola en los Andes. 


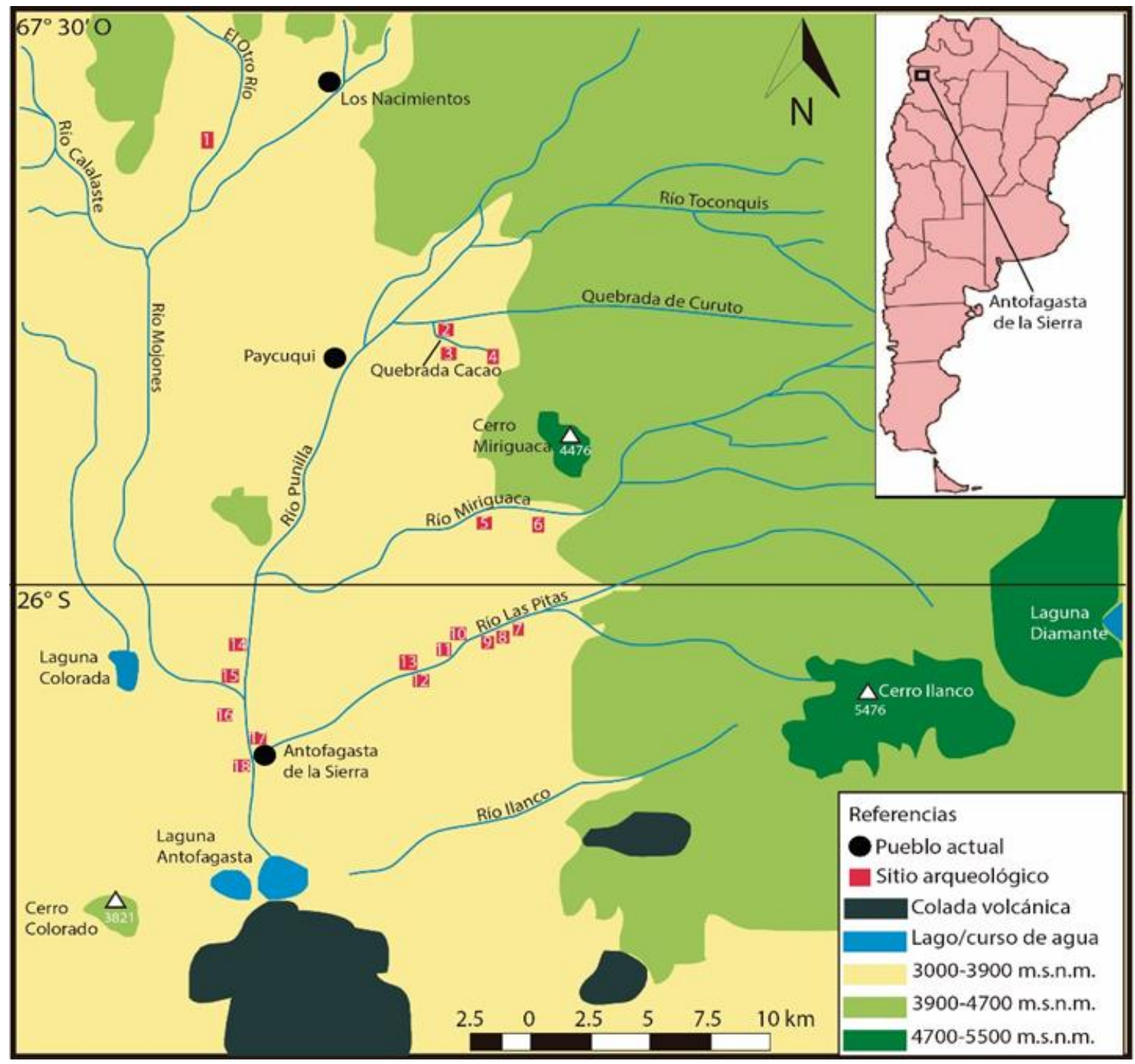

Figura 1. Distribución de los sitios con arte rupestre formativo en ANS. 1. Corral Grande $1 ; 2$. Curuto 5; 3. Cueva Cacao 1; 4. Cueva Cacao 3; 5 . Sector Intermedio río Miriguaca; 6. Campo de las Tobas; 7. El Sembrado; 8. Piedra Horadada 2; 9. Punta de la Peña; 10. Peña de las Trampas; 11. Peñas Chicas; 12. Peñas Coloradas; 13. Barda Alta del Río las Pitas; 14 Casas Viejas; 15. Confluencia; 16. Derrumbes; 17. Punta del Pueblo; 18. La Torre.

\section{El Formativo en Antofagasta de la Sierra}

La Puna Argentina presenta evidencias de ocupación desde hace más de 10000 años $\mathrm{AP}$, cuando sociedades cazadoras-recolectoras ocuparon el área y explotaron sus recursos. A partir de $c a .4500$ años AP la evidencia señala un proceso creciente de circunscripción territorial, aumento del sedentarismo y una incipiente transición de la caza-recolección hacia el pastoreo de camélidos (Aschero y Hocsman 2011). 
Las investigaciones enfocadas en los momentos posteriores a 3000 años AP demostraron que para este momento las sociedades puneñas comenzaron un proceso de sedentarización que incluyó estrategias productivas mixtas basadas en el pastoreo y la agricultura (Olivera et al. 2004). La evidencia paleoclimática sugiere la existencia de un período húmedo entre ca. 3000-1600 años AP, el cual favoreció el asentamiento en aldeas y la intensificación de economías productivas. Asimismo, se aprovechó de manera integral la oferta diferencial de recursos correspondientes a distintos sectores ambientales, como propone el Modelo de Sedentarismo Dinámico (Olivera 1992). Según este modelo, las Quebradas de Altura (3900-4600 msnm) fueron explotadas para actividades de caza y pastoreo mientras que en el Fondo de Cuenca (3400-3500 msnm) se instalaron sitios de residencia permanente en los cuales se realizaron actividades productivas. Los Sectores Intermedios (3550-3900 $\mathrm{msnm})$, por otro lado, se aprovecharon de forma menos intensiva, dado que los recursos presentes en este sector son menores en comparación con los descritos anteriormente. Aun así, hacia ca. 2000 años AP, hay evidencia de ocupaciones más intensivas y permanentes en los Sectores Intermedios, en concordancia con un contexto climático de creciente aridización (Olivera et al. 2004).

Debemos agregar a esta síntesis las evidencias de arte rupestre en la microrregión, una manifestación importante de la vida de los grupos que ocuparon ANS desde el Período Arcaico en adelante. Aschero (1999) desarrolló una secuencia cronológica relativa a las representaciones rupestres, en la cual sostiene que las modalidades estilísticas que corresponden al Período Formativo muestran figuras de máscaras, llamas felinizadas, caravanas de llamas, figuras humanas con tocado y figuras de camélidos bicápites y cuadricápites, entre otros motivos. En cuanto a su distribución geográfica, Martel (2010) remarca que las representaciones rupestres asignadas al Formativo tienden a concentrarse en las Quebradas Intermedias, entre 3550 y 3900 msnm (Figura 1), lo cual se condice con el rol que tuvieron estos sectores en la subsistencia de los grupos humanos durante este período. En este sentido, ya fue advertido por otros autores que, asociados a muchos de los sitios de residencia y explotación de recursos, se encuentran motivos tallados o pintados en la roca, lo cual habría implicado que el arte rupestre estaba integrado al sistema general de asentamiento-subsistencia de la microrregión (Olivera y Podestá 1993; Martel 2006). Esto indicaría una relación entre el arte rupestre formativo y actividades de índole productiva para la microrregión.

Por último, queremos hacer mención del papel que se ha propuesto para algunas representaciones como marcadores territoriales, las cuales delimitaban el acceso a espacios de pastoreo de camélidos domesticados y tierras aptas para los cultivos y, de esta forma, aseguraban la explotación exclusiva de los recursos naturales de cada sector a los grupos sociales (familias o linajes) que los ocupaban (Aschero 2007). 
Corral Grande 1 y su entorno ambiental

El sitio Corral Grande 1 (de ahora en más CG1) donde se localiza el arte rupestre que es objeto de este análisis, se ubica a $22 \mathrm{~km}$ al norte de la localidad de Antofagasta de la Sierra, sobre la terraza del Otro Río, un afluente del río Mojones, a $3730 \mathrm{msnm}$, en los Sectores Intermedios de la microrregión. Se trata de un asentamiento multicomponente cuya evidencia, tanto artefactual como arquitectónica, permite relacionarlo con ocupaciones que abarcan desde el Período Formativo hasta momentos históricos, pasando por el Período Tardío (Olivera et al. 2015).
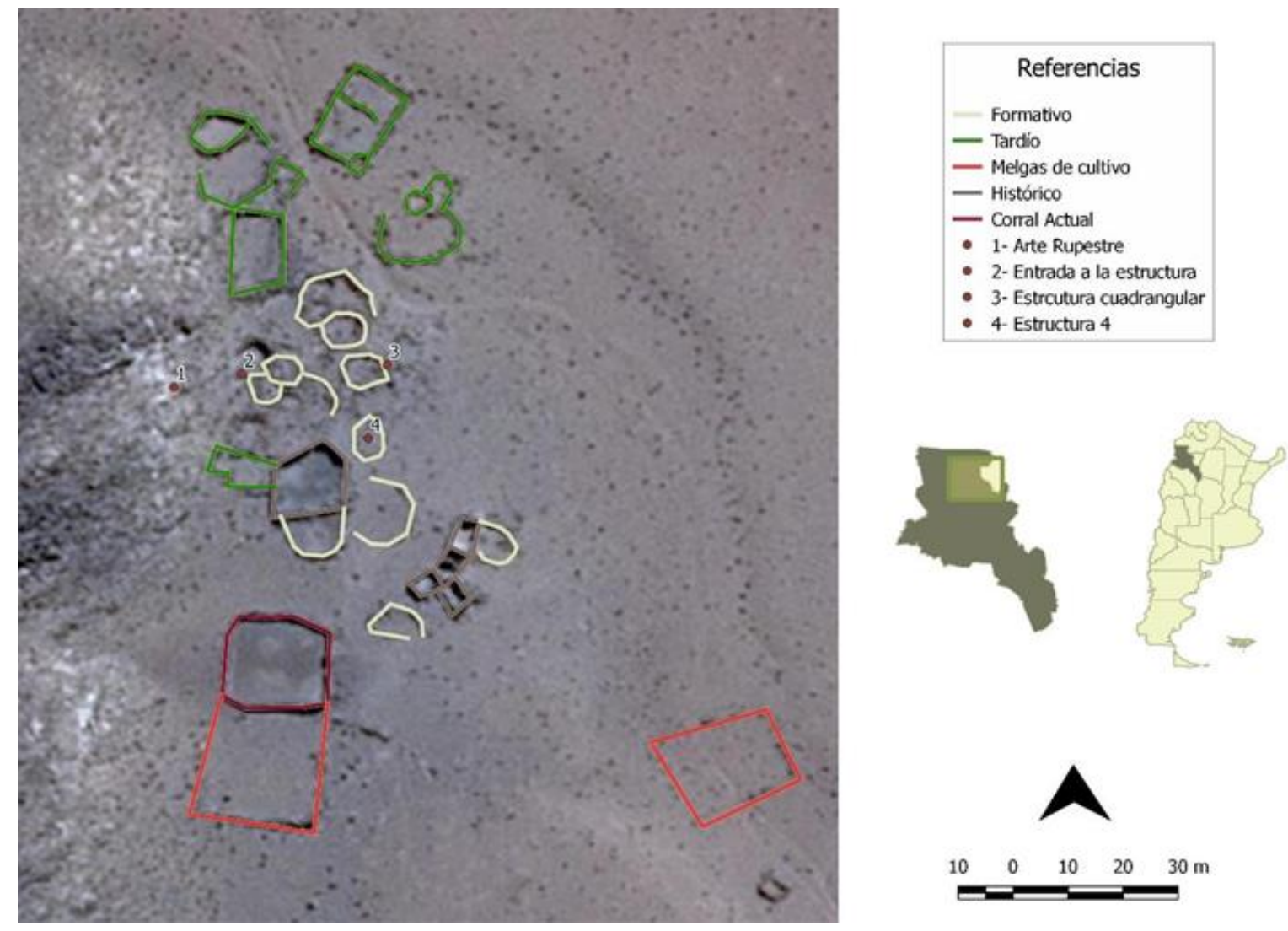

Figura 2. Plano del sitio Corral Grande 1 con las estructuras correspondientes a diferentes períodos cronológicos marcadas. Tomado y modificado de Olivera et al. 2015.

En los primeros momentos de ocupación podemos ubicar a las estructuras circulares (Figura 2). Los materiales de superficie y aquellos provenientes de un sondeo en la estructura 4 apoyan esta interpretación, particularmente los estilos cerámicos adscriptos a momentos formativos (Saujil, Ciénaga y Aguada) hallados dentro de la estructura. Estos materiales señalarían una ocupación formativa tardía, es decir, posterior a ca. 2000 AP (Olivera et al. 2015). Esto se condice con la propuesta de una ocupación más intensa de los sectores intermedios en la microrregión durante este período. Además, una datación radiocarbónica sobre carbón proveniente de la 


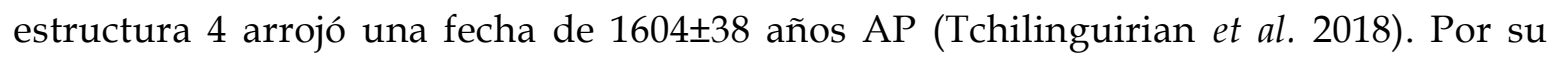
parte, las estructuras de planta rectangular habrían pertenecido a momentos Tardíos, ca. 1000 años AP, mientras que hay claras evidencias de estructuras tanto históricas como actuales (específicamente corrales).

Considerando el arte rupestre en particular, este fue hallado sobre dos bloques de basalto que sirven como base a tres paneles en que están representados siete motivos grabados. Dichos bloques de roca se ubican en la falda oriental del cerro en cuya base se emplaza el sitio arqueológico CG1. El grado de conservación del arte rupestre en el sitio no es óptimo, ya que el soporte y los motivos presentan erosiones de carácter natural. Incluso, un tercer bloque muestra rastros de posibles representaciones que no pudieron ser definidas debido al alto grado de alteraciones naturales (Figura 3).

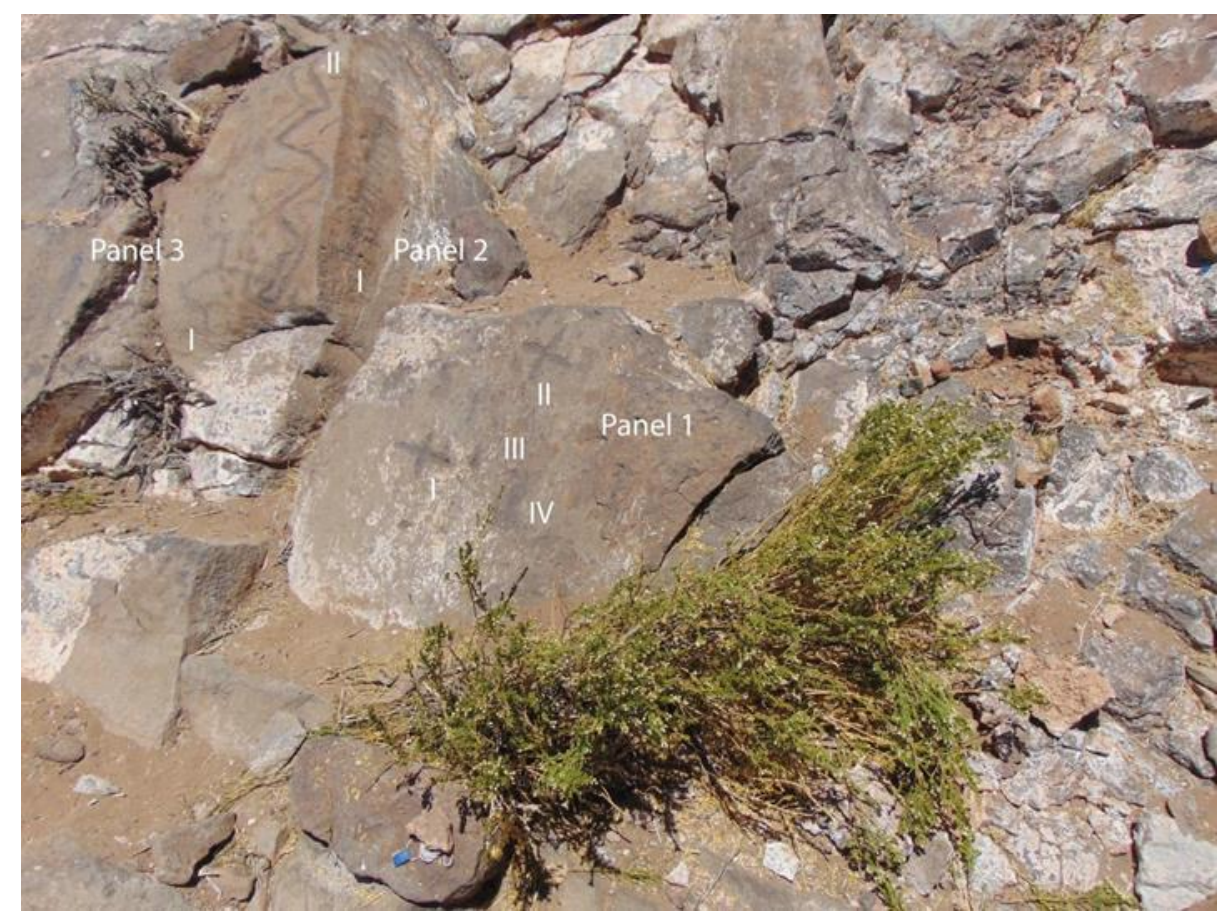

Figura 3. Disposición de los tres paneles registrados y los respectivos motivos

En cuanto al ambiente que domina el sector de emplazamiento de CG1, una reconstrucción de la paleogeografía pluvial del área indica que entre ca. 2000-1800 años AP la cuenca del río Mojones, incluyendo sus afluentes, era de régimen permanente, con una llanura de inundación con suelos orgánicos y una vega que presentaba buenos recursos de forraje. Esto se condice con las condiciones generales de mayor humedad que se presentaron en toda el área durante el Formativo. En momentos posteriores (1800-300 años $\mathrm{AP})$, la paleovega se degradó y retrotrajo aguas arriba, con su consecuente disminución de recursos disponibles. Si bien durante un breve lapso en momentos históricos (300-240 años $\mathrm{AP})$ el río Mojones volvió a ser de régimen permanente, en la actualidad se presenta como 
un curso de agua efímero e inestable, con suelos de escaso desarrollo (Tchilinguirian et al. 2018). El paisaje que hoy en día circunda a CG1, está dominado por la vega formada en torno al curso del Otro Río, actualmente muy degradada y de régimen intermitente.

Estos cambios en el río y la vega no sólo afectaron la disponibilidad de recursos, si no que parecen haber impactado de manera casi directa sobre la toma de decisiones de las poblaciones locales. La expansión de la vega durante el Formativo habilitó una ocupación demográficamente mayor y con un ciclo de permanencia estable en el sitio, lo cual se ve reflejado en los restos materiales obtenidos del sondeo: las evidencias lítica y cerámica indican una gran cantidad de actividades de procesamiento y/o consumo, lo cual estaría relacionado con un contexto doméstico (Olivera et al. 2015). A medida que avanzó la degradación de la vega, la disminución en la disponibilidad de recursos pudo haber generado una menor intensidad en la ocupación del espacio.

\section{Metodología y resultados}

Para realizar el presente trabajo tomamos como unidades de análisis a los motivos. Seguimos a Aschero et al. (2003) a la hora de distinguir entre representaciones y motivos. De acuerdo con estos autores, ambos son conceptos que operan en diferentes pasos del estudio del contenido de un sitio con arte rupestre. Las representaciones consisten en la visualización de las unidades discretas, es decir aisladas en el espacio, que fueron producidas por un único gesto técnico completo, mientras que el motivo tiene que ver con las articulaciones que es posible distinguir entre representaciones, ya sean por inclusión, vínculos anecdóticos, etc. Por lo tanto, al tomar al motivo como unidad de análisis, las representaciones se constituyen como los elementos que forman al motivo. Esta distinción nos permitirá indicar el número mínimo de operaciones técnicas originalmente ejecutadas sobre la pared, lo cual da cuenta del grado de cobertura de un soporte, hecho especialmente importante cuando el número de motivos es bajo.

En campo se realizó el relevamiento de los grabados. En primer lugar, se identificaron las representaciones y los motivos a los que dan lugar. En cada uno de los tres paneles, estos fueron individualizados e identificados con número romanos. Posteriormente se procedió al registro de las figuras. A través del calco, se tomaron las medidas del ancho y el largo máximo. Asimismo, se documentaron los paneles con fotografías con más de un $90 \%$ de superposición, las cuales fueron utilizadas para generar un modelo 3D a partir de fotogrametría, con el uso del software Agisoft Photoscan Standard. Las fotografías fueron tomadas con una cámara fotográfica Sony DSC-H300 de 20.1 Mega Pixeles.

De esta manera, se registró un total de 20 representaciones y siete motivos (Tabla 1). En todos los casos las representaciones se lograron mediante la técnica de picado plano. El grado de pátina que recubre a todos los motivos analizados es el mismo, aún en los paneles con distinta orientación. Ello indica un único evento de ejecución o ejecuciones en eventos con muy poca separación temporal entre sí. 
No se registraron superposiciones en los bloques analizados. Esto es de especial interés si tenemos en cuenta la evidencia de reocupaciones en el sitio habitacional. Además de no haber modificaciones o intentos de obliterar los motivos, tampoco aparecen otros grabados en ninguno de los bloques presentes en el cerro, el cuál fue prospectado en busca de más motivos durante la campaña de 2018. Esto significa que no sólo no se cubrieron los motivos tempranos a lo largo de las reocupaciones posteriores, sino que tampoco se generaron nuevas representaciones. La presencia de estos bloques durante ocupaciones posteriores a su elaboración resulta de particular interés respecto de las relaciones que los habitantes de los períodos Tardío y Colonial habrían tenido con los mismos y el por qué de la decisión de no superponer ni agregar nuevos motivos.

\begin{tabular}{|l|c|c|c|}
\hline & \multicolumn{2}{|c|}{ Bloque 1 } & Bloque 2 \\
\hline & Panel 2 & Panel 3 & Panel 1 \\
\hline Orientación & Norte & Este & Este \\
\hline Total de representaciones & 6 & 3 & 11 \\
\hline Total de motivos & 1 & 2 & 4 \\
\hline Motivos figurativos & 1 & 1 & 2 \\
\hline Motivos no figurativos & - & 1 & 2 \\
\hline
\end{tabular}

Tabla 1. Detalle de disposición de los motivos y representaciones en los paneles analizados.

El panel 1, de $87 \mathrm{~cm}$ de ancho por $62 \mathrm{~cm}$ de alto, se orienta hacia el este y está constituido por una única cara de una roca, mientras que los otros son dos caras de una misma roca, una de ellas orientada al norte, de 1,05 m de ancho por $21 \mathrm{~cm}$ de alto (panel 2), y otra al este, de $80 \mathrm{~cm}$ de alto por $43 \mathrm{~cm}$ de ancho (panel 3). Cada uno de los paneles fue tomado como una unidad topográfica de relevamiento (UT).

En el panel 1 se pudieron identificar dos motivos de cruces simples de brazos iguales, una huella de felino y una pisada humana (Figura 4). Las dos cruces presentan dimensiones similares: la I es de $11 \mathrm{~cm}$ de alto por $12 \mathrm{~cm}$ de ancho y la II es de $9 \mathrm{~cm}$ de alto por $12.5 \mathrm{~cm}$ de ancho, mientras que la pisada de felino mide $7 \mathrm{~cm}$ de alto por $5,5 \mathrm{~cm}$ de ancho y la pisada humana $8 \mathrm{~cm}$ de alto por $6 \mathrm{~cm}$ de ancho. Ambos motivos de cruces presentan una buena preservación y se pueden observar claramente a simple vista, mientras que la huella de felino y la pisada humana $8 \mathrm{~cm}$ de alto por $6 \mathrm{~cm}$ de ancho. Ambos motivos de cruces presentan una buena preservación y se pueden observar claramente a simple vista, mientras que la huella de felino y la pisada humana muestran 
un alto grado de deterioro, característica que dificultó la identificación de estos motivos en el terreno. Esta diferencia de preservación en el mismo bloque puede deberse al carácter irregular de la superficie de la roca.

Las figuras de cruces aparecen en otros sitios de ANS. En el sitio Curuto 5, ubicado en la quebrada de Curuto, este motivo se asocia con figuras humanas, de camélidos y suris, todas hechas mediante la técnica de picado (Aschero et al. 2003). Por asociación con otros motivos, las cruces se pueden relacionar con la modalidad Peñas Chicas definida por Aschero (1999), ubicada cronológicamente entre 1700 y 1500 años AP, un momento en que se dio una integración plena entre la agricultura y el pastoreo de camélidos. Motivos de este tipo asociados al Período Formativo fueron también hallados en la quebrada del río Miriguaca (Martel y Escola 2011).

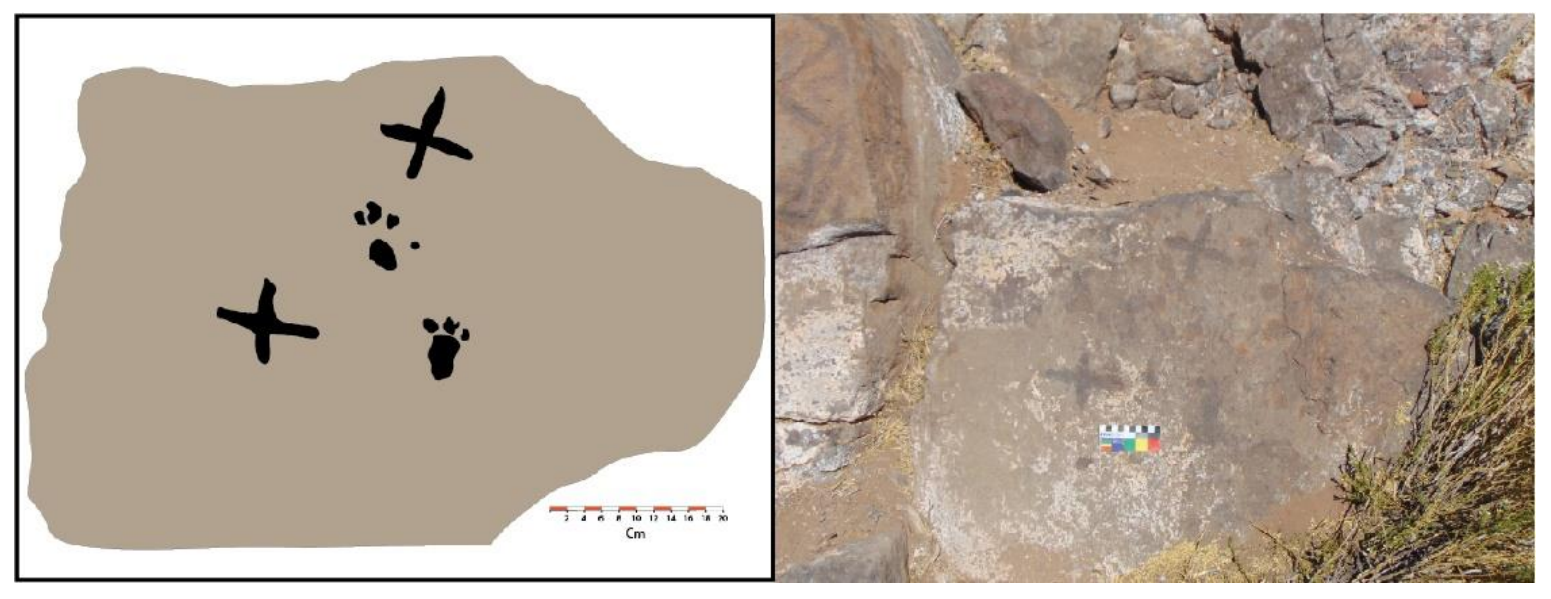

Figura 4. Sitio Corral Grande 1. Panel 1. Figuras de cruces, huella de felino y pisada humana.

La huella de felino está constituida por cinco elementos: cuatro improntas de dedos y la almohadilla principal. Este tipo de motivo aparece en varios sitios de ANS, tanto en el Fondo de Cuenca, en los sitios Confluencia y Punta del Pueblo, como en las Quebradas Intermedias, en los sitios Campo de las Tobas y Peñas Coloradas (Figura 9). En Confluencia, un complejo de sitios de carácter ritual (Aschero 2007), entre los cuales los motivos de arte rupestre representados abarcan desde el Período Arcaico hasta momentos de contacto europeo, Martel et al. (2012) registraron 11 huellas de felino, las cuales fueron asignadas tanto al Período Formativo como al Tardío. Por otro lado, el sitio Campo de las Tobas, con una cronología entre 2400-1300 AP (Podestá et al. 1991) presenta, a su vez, huellas de felinos entre otros motivos. Estilísticamente, la huella de felino de CG1 es similar a la mayoría de los casos observados en Confluencia por Martel et al. (2012) y en Campo de las Tobas por Podestá et al. (1991) y Podestá y Falchi (2015). En ambos sitios la mayoría de estos motivos están compuestos por cinco elementos al igual que en CG1. Los casos atípicos, aquellos en que las improntas de dedos representadas son cinco o más, son minoritarios en los dos sitios. 
Por último, en este panel destaca el motivo de la pisada humana. Al igual que en el caso de las huellas de felino, este tipo de motivo aparece en los sitios Confluencia, Campo de las Tobas y se agregan Cueva Cacao y Barda Alta del Río las Pitas (Figura 9). Pero es en Campo de las Tobas donde fue registrada la mayor cantidad de pisadas humanas $(\mathrm{N}=16)$, reconociéndose una variabilidad tipológica en el sitio (Podestá et al. 1991; Falchi y Podestá 2015). Si comparamos estilísticamente la pisada humana de CG1, observamos que es característico de este sitio la impronta de solo tres dedos ejecutada mediante picado. En la mayor parte de los casos analizados en otros sitios, las huellas humanas presentan improntas de cuatro o cinco dedos. Resulta interesante remarcar que la asociación que registramos en este panel entre pisadas humanas y huellas de animales, específicamente de felino, ya ha sido observada en otros sitios de la microrregión (Falchi y Podestá 2015).

Si bien el inicio del proceso de domesticación de camélidos en la Puna de Atacama (que abarca territorios del norte de Chile, sur de Bolivia y el noroeste de Argentina) se remonta a 6000/5000 años AP (Olivera 1998), la representación de llamas en diferentes actitudes y escenas comienza a aparecer con frecuencia en la Puna Sur Argentina recién a partir de circa 2500 años AP (Aschero 2000). Una de las escenas en las cuales fueron figuradas son alineaciones que representan caravanas, a veces dirigidas por un personaje humano. Este es el caso del único motivo representado en el panel 2 de CG1. Se trata de una caravana compuesta por cinco camélidos y un personaje guía, de $58 \mathrm{~cm}$ de ancho y orientada al oeste. Al igual que el resto de los motivos del sitio, la técnica de ejecución consistió en picado plano (Figura 5).

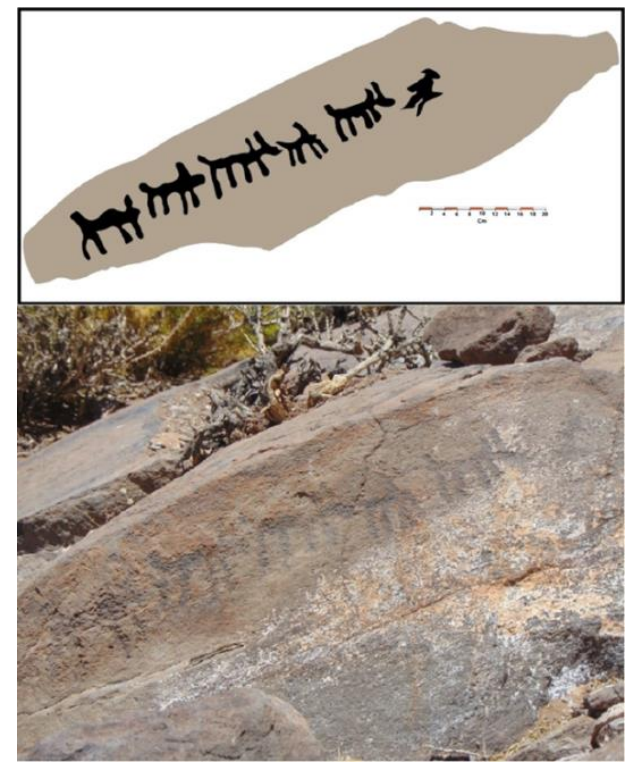

Figura 5. Sitio Corral Grande I. Panel 2. Motivo de caravana de camélidos 
Los camélidos fueron representados de perfil, con cuatro patas, cola y orejas. Este patrón de diseño es similar a otros motivos de caravana presentes en el área, adscriptos a la modalidad Peñas Chicas (Aschero 1999). Motivos de camélidos similares a los aquí analizados aparecen en el llamado Bloque Caravanas en la Quebrada de Miriguaca (Martel y Escola 2011), en el sitio Derrumbes en el Fondo de Cuenca y en los sitios Punta de la Peña 13 y Peñas Coloradas 1.2 en la Quebrada de Las Pitas (Figura 9), entre otros (Martel 2010).

La presencia de este motivo en el sitio, en relación con modelos propuestos para momentos formativos en el área (Olivera 1992; Olivera et al. 2015), nos lleva a pensar que CG1 podría haber sido un espacio donde se llevaban a cabo actividades vinculadas a la práctica caravanera. Esta idea será desarrollada en mayor profundidad más adelante.

En el último panel, el 3, observamos dos motivos. El primero de ellos consiste en una figura antropomorfa masculina, con el sexo claramente marcado, con los brazos en alto y las piernas abiertas, de $30 \mathrm{~cm}$ de alto por $26 \mathrm{~cm}$ de ancho. Este motivo nos recuerda a la figura femenina contenida en el interior de un camélido bicápite descripta por Aschero (2006 y 2007) en el sitio Confluencia (Figura 9), a pesar de que presenta algunas diferencias formales. Ambas representaciones muestran deliberadamente el sexo del personaje (masculino en CG1 y femenino en Confluencia) y la postura de los antropomorfos es similar, aunque difieren en la posición de los brazos. Respecto de este último aspecto, figuras humanas con las manos alzadas aparecen frecuentemente representadas en el ámbito andino centro-sur, en ambas vertientes de la cordillera, para momentos del Formativo tardío (Aschero 2007).

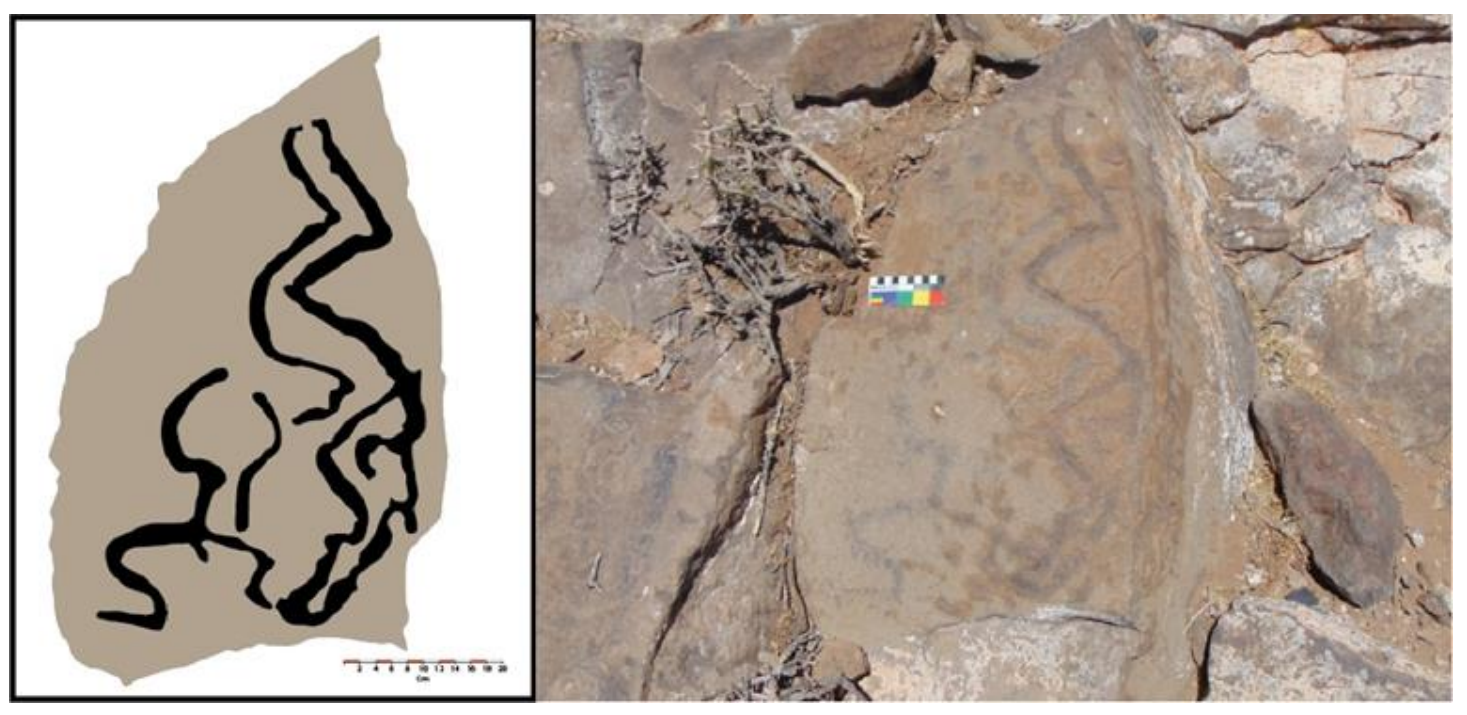

Figura 6. Sitio Corral Grande I. Panel 3. A la izquierda, figura antropomorfa de las manos alzadas. A la derecha, figura lineal en forma de zig-zag. 
El segundo motivo del panel, ubicado a la derecha de la figura antropomorfa, se trata de una representación lineal en zig-zag: un conjunto de grabados integrados por surcos curvilíneos, de $68 \mathrm{~cm}$ de alto por $21 \mathrm{~cm}$ de ancho, ejecutados mediante picado plano (Figura 6). Con el objetivo de individualizarlo, le daremos a este motivo el nombre de sistema de riego, ya que lo consideramos una de las variantes de representación de maqueta que se hallan en ANS para momentos Formativos y Tardíos (Aschero et al. 2009). Motivos similares a este fueron hallados en los sitios Piedra Horadada 2, Punta de la Peña 4, Punta de la Peña 9 y El Sembrado (Figura 9). A partir de la asociación de motivos, análisis de pátinas y dataciones radiocarbónicas, Aschero et al. (2009) ubicaron cronológicamente a los motivos de sistema de riego en el Período Formativo, específicamente en la modalidad Peñas Chicas.

En relación con este motivo, queremos señalar la similitud que muestra con otros motivos hallados en ANS, respecto de su emplazamiento próximo a un curso de agua permanente y de importante potencial agrícola (Aschero et al. 2009). Debemos recordar que durante el Período Formativo el ambiente de la microrregión era más húmedo que en la actualidad y por lo tanto la vega cercana a CG1 era un curso de agua permanente (Tchilinguirian et al. 2018). Este dato es importante porque nos permite un acercamiento al significado del motivo, cuestión que será abordada en el siguiente apartado.

\section{La representación del agua en un asentamiento agrícola pastoril}

Muchos de los motivos representados por las sociedades andinas desde tiempos prehispánicos se relacionaron con la percepción que tenían dichos pueblos de su relación con el mundo circundante. El entorno y los ciclos anuales marcaban el ritmo de las actividades agrícolas y el pastoreo de camélidos (Joslyn 2013). En este sentido, el arte rupestre, no debería ser entendido únicamente como objeto estético, dado que no es solo producto de la sensibilidad humana, sino que además está imbricado en una compleja interacción de factores objetivos y extraestéticos, como las prácticas sociales y económicas de un grupo humano (Espinosa 1996). Por lo tanto, nos preguntamos ¿cuál es el sentido de la representación en zig-zag de un sistema de riego en un asentamiento de pastores en que la agricultura tenía un papel menor?

En primer lugar, debemos tener en cuenta que la representación de rasgos del paisaje circundante mediante grabados en roca es una práctica que ya ha sido observada en los Andes, como el caso analizado por Lane et al. (2018) en los Andes Centrales, en el sitio ABL 1 en la Quebrada de Ablomé, Salta (Falchi y Podestá 2019), y en los casos de las maquetas citados anteriormente para otros sitios de ANS (Aschero et al. 2009). De este modo, el motivo aquí analizado pudo haberse tratado de una reproducción del curso de agua (vega) que en el pasado fluyó de forma continua a través de los campos próximos a CG1, irrigando las tierras para el pastoreo y la agricultura. Al mismo tiempo, la forma del motivo en zig-zag puede tratarse de una representación asociada a otros aspectos de la 
vida de quienes habitaron la región en el pasado. En la cosmología Andina, la representación del zig-zag era una alusión a la serpiente y al rayo (Quiroga 2017 [1929]). De acuerdo con Seibold (1992), quien trata con los símbolos representados en textiles en Perú, los zig-zags geométricos fueron una forma de representar el agua en movimiento, los relámpagos y las serpientes. En este último caso se trata de la simbolización de un animal que trae el agua, evento de gran importancia para la agricultura en las regiones andinas áridas. La serpiente entraba dentro del dominio de Ukhu Patsa, el cual refería al mundo de abajo, junto con los ancestros prominentes y el agua subterránea. En los Andes Centrales, por ejemplo, el motivo de la serpiente surgiendo de la roca representa la conexión entre dos mundos: el plano de la tierra, el mundo físico como lo conocemos (Kay Patsa) y el mundo de abajo (Ukhu Patsa), agua subterránea que emerge a la superficie (Ambrosino 2017).

Si nos concentramos en la importancia del agua para las actividades agrícolas en los Andes, estas fueron acompañadas de un conjunto de ofrendas y rituales de carácter propiciatorio, en los cuales se movilizaba a las fuerzas de la naturaleza, a los antepasados y a las fuerzas sociales del ayllu. En los relatos míticos andinos, las divinidades y los antepasados participaron en la acción de canalizar y encausar artificialmente el agua (de deshielo, de manantiales, de lagos, etc.). En algunos de estos mitos, los fundadores legendarios de las obras de canalización se convirtieron en rocas ubicadas en la proximidad de las vertientes (Aschero et al. 2009) e incluso en la actualidad, estas rocas cobran relevancia en el curso de las ceremonias que inauguran la estación de riego, mediante las cuales las comunidades piden por la abundancia de agua para los cultivos (Castro y Varela 1994). En la región de la Puna de Atacama, específicamente en el Norte de Chile, los pobladores locales denominan a los motivos de chacras grabados en las rocas como "chacras de los antiguos gentiles" y los incorporan a actuales rituales agrícolas (Briones et al. 1999). De esta manera, las representaciones de sistemas de riego y de chacras en la roca pueden haber formado parte de rituales que llevaron a cabo las sociedades prehispánicas, desplazando el agua artificialmente en el marco de un simbolismo propiciatorio.

De esta forma, consideramos que el caso del motivo de sistema de riego del panel 3 podría estar vinculado con este tipo de rituales propiciatorios de fertilidad agrícola y productiva. Esta interpretación se basa en las características morfológicas y estilísticas del motivo, en el lugar de emplazamiento del sitio, como ya adelantáramos, en el material hallado en excavación y en la relación espacial que tiene el soporte con rasgos arquitectónicos adyacentes. Si se mira desde el punto en que se ubican los bloques con arte rupestre en dirección este, se puede observar que estos están alineados con el acceso, dirigido directamente hacia el oeste, a una estructura circular adscripta al Período Formativo y a una mayor distancia, con una plataforma cuadrangular hecha con rocas (Figuras 7 y 8). Si bien no podemos afirmar que haya un vínculo de tipo ritual en base a esta relación espacial, creemos que sería un trabajo fructífero explorar este tipo de asociación entre el arte rupestre y la arquitectura del sitio, especialmente con la plataforma cuadrangular. 


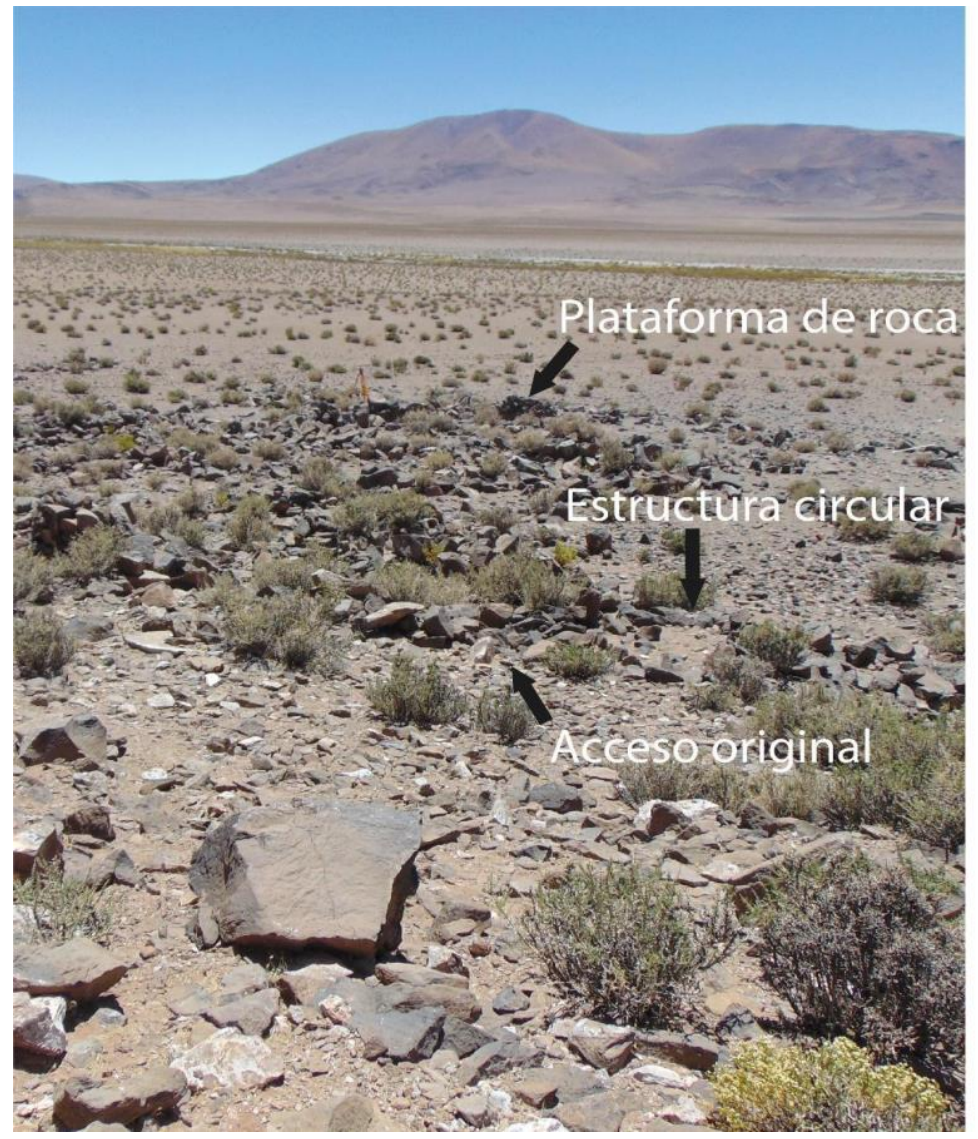

Figura 7. Vista de la estructura circular y la plataforma de roca desde el bloque con arte rupestre. Dirección oeste-este. En el fondo se observa la vega actual.

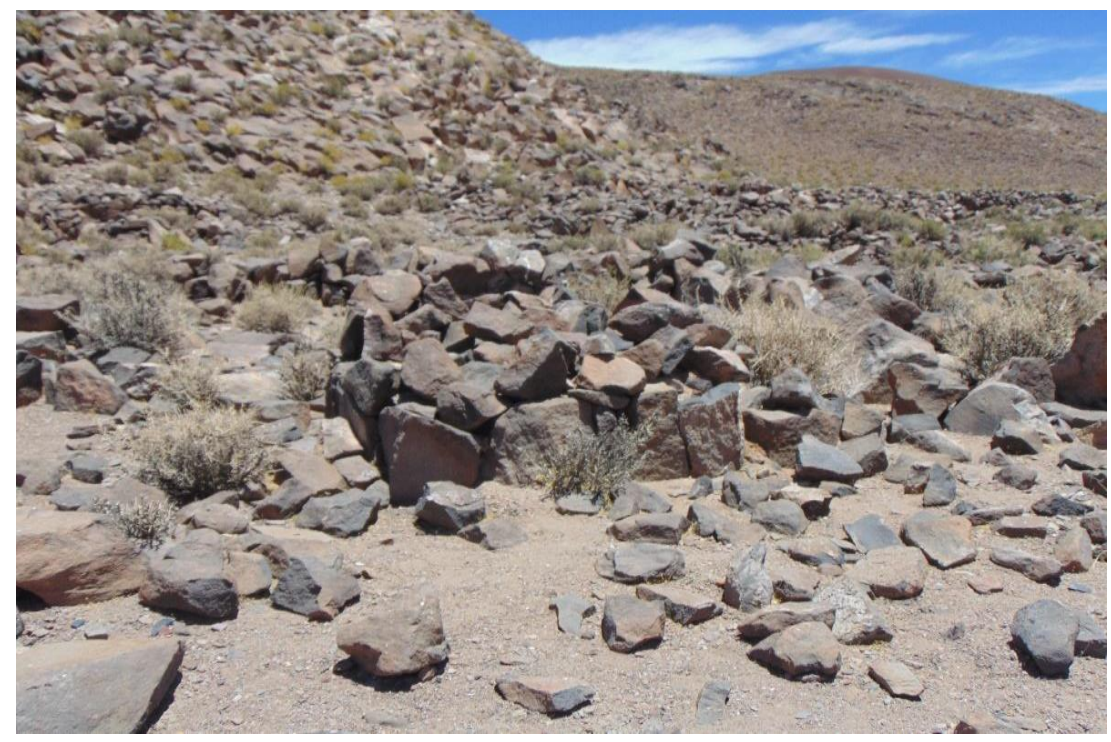

Figura 8. Estructura rectangular. 
El material hallado durante las excavaciones de 2018 en el sitio puede ser interpretado como apoyo a esta propuesta ya que la información obtenida del análisis del mismo da cuenta de la explotación de llamas, demostrando la presencia de animales domesticados (Grant et al. 2019). A ello se suma la presencia de dos melgas pircadas en el sitio (Figura 2), junto con el hallazgo de una pala lítica en estratigrafía, evidencia que indica la realización de actividades agrícolas en pequeña escala. La ausencia de canales que transportaran agua desde la vega hacia las melgas es indicio de que el agua para regadío era obtenida directamente desde el curso de agua. Ello refuerza la importancia de este rasgo del paisaje en las actividades productivas desarrolladas en CG1, teniendo en cuenta que, en una región tan árida como ANS, el agua es de vital importancia para la explotación agrícolapastoril, aún si la misma se hubiese dado en pequeña escala.

Además, no debemos olvidar la asociación de este motivo con la figura antropomorfa de los brazos en alto que se halla en el mismo panel. Como anteriormente observamos, una figura femenina estilísticamente similar y asociada a la imagen de un camélido de dos cabezas (Figura 9) fue analizada por Aschero (2006 y 2007), quien considera que un posible significado es el de la femineidad como mediadora en el proceso de domesticación de camélidos, en tanto la figura de la mujer podría estar involucrada en la domesticación de plantas y animales dada su imagen de fertilidad, nutrición y cuidado de su progenie. Aunque consideramos que dicha interpretación debe ser tomada con cuidado, dado que se desconoce el rol asignado a las mujeres durante el Período Formativo en las sociedades puneñas, nos parece interesante la idea de la relación entre los personajes representados y la fertilidad, sobre todo al tener en cuenta el énfasis buscado en la representación de los genitales. ¿Podríamos considerar que la figura masculina en CG1 sea una representación de la fertilización masculina de las tierras para el cultivo mediante el agua que fluye desde los cerros más altos? A este respecto, hay que tener en cuenta la percepción Andina de los ríos que proceden de las montañas como de origen masculino (Aschero et al. 2009). Creemos que vale la pena en el futuro profundizar acerca del significado de este motivo y su asociación con el resto de las figuraciones del sitio.

De esta forma, vemos que el motivo en el que nos centramos en este apartado podría representar al agua que fluía por la vega que se encuentra a aproximadamente 180 metros de distancia del sitio de habitación. Al mismo tiempo, podría llevar inscripta una alusión al rayo o la serpiente, como se explicó con anterioridad. Ambas significaciones se solaparían en una forma ritual de pedir por la abundancia de agua y, en definitiva, la proliferación de los cultivos y de pasturas para el pastoreo. Esta interpretación debe ser entendida dentro del sistema económico de las sociedades puneñas del Período Formativo, cuya base para la subsistencia era principalmente el pastoreo, con el complemento de la caza de camélidos y una agricultura de baja intensidad (Olivera 1992). 


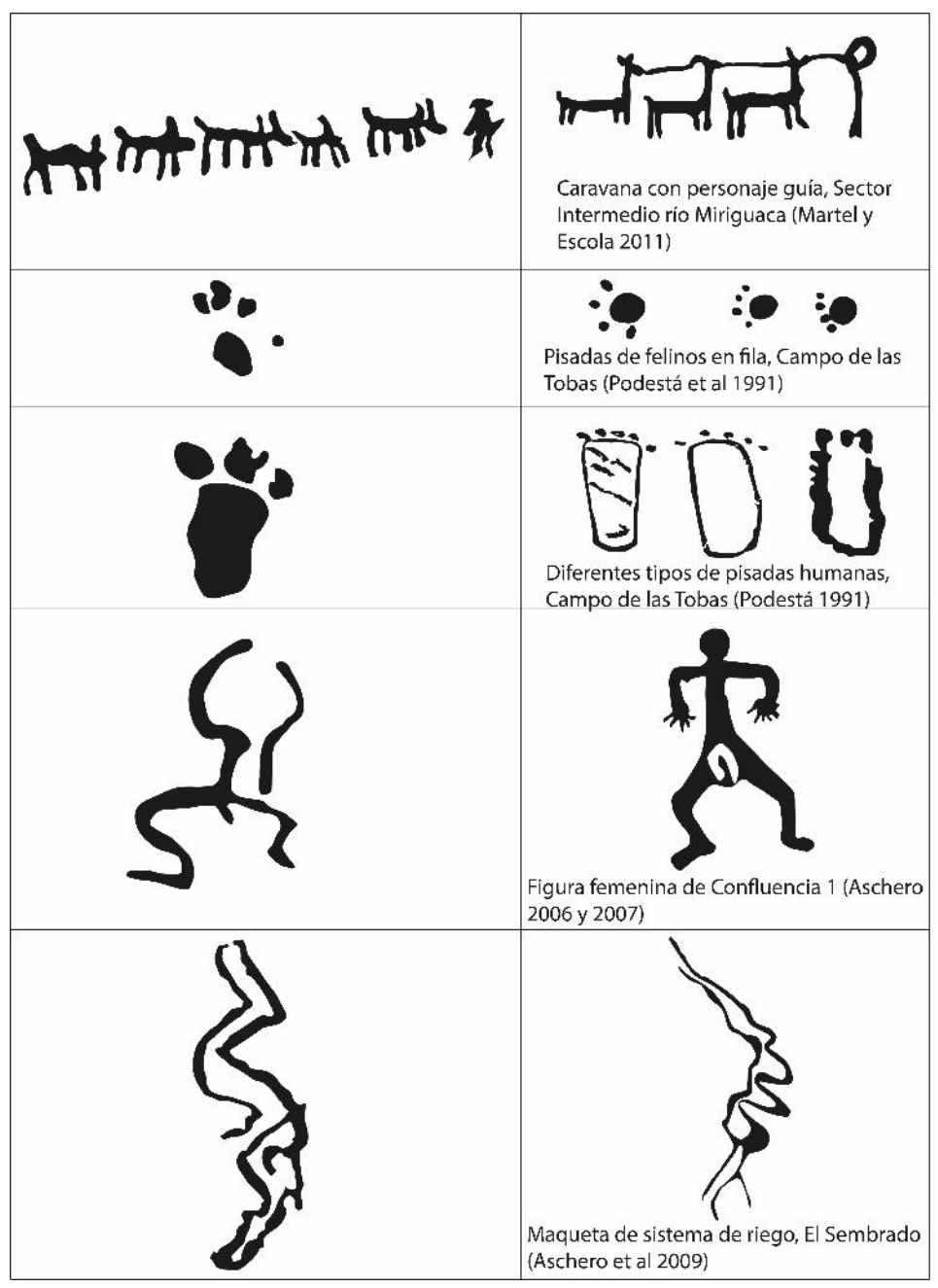

Figura 9. Comparación entre los motivos de CG1 (columna izquierda) y otros provenientes de distintos sitios de ANS (columna derecha)

\section{Conclusiones}

El primer punto que podemos abordar desde el análisis llevado a cabo es de orden cronológico. Basándonos principalmente en dos de los motivos analizados, la caravana de llamas con personaje guía y la representación interpretada como un posible sistema de riego, sumado a los motivos asociados a las cruces en otros sitios, podemos asignar el arte rupestre de CG1 al Período Formativo, más precisamente a la modalidad Peñas Chicas. Esta asignación cronológica es coherente con las periodificaciones anteriormente asignadas al sitio residencial, con base en la cerámica hallada en superficie y en un sondeo, así como con el tipo de estructuras (Olivera et al. 2015). Sin embargo, nos permite indirectamente afinar más el rango cronológico del sitio dentro del Período Formativo ya que, como mencionáramos antes, Peñas Chicas corresponde cronológicamente a 1700-1500 años AP. $\mathrm{Si}$ bien algunos motivos típicos de esta modalidad, como enfrentamientos o figuras 
armadas o con tocados, están ausentes, por el momento y hasta tener mayor evidencia asociada en el sitio, sostenemos la pertenencia a la misma de los grabados presentados en este trabajo. Por otra parte, no fue posible identificar motivos que sean asignables a los períodos Tardío y Colonial, a pesar de que la arquitectura del sitio indica una ocupación durante ambas épocas. Aun así, hay que tener en cuenta que el motivo de la huella del felino en el sitio podría ser asignado tanto al Formativo como al Tardío, así como también las cruces simples. Sin embargo, la homogeneidad en la pátina respecto de los otros motivos nos hace pensar en una misma temporalidad para todos los motivos.

En segundo lugar, advertimos que el arte rupestre de CG1 repite características que se dan a nivel regional. Por un lado, respecto de su distribución geográfica, como ya anticipamos, Martel (2010) señala que el arte rupestre durante el Período Formativo aparece más concentrado en los Sectores Intermedios (3550-3800 m.s.n.m.), situación que cambia durante el Tardío, época en la que suele concentrarse en el Fondo de Cuenca (34003550 m.s.n.m.). Por otro lado, en ANS el arte rupestre estuvo asociado a espacios domésticos y a sectores propicios para el pastoreo, la caza y la agricultura. Ya fue indicado por otros autores (Olivera y Podestá 1993; Martel 2006) que en ANS los sitios con figuras pintadas o talladas en la roca se encuentran vinculados a estrategias de explotación de los recursos y por ello integrados al sistema de asentamiento-subsistencia de la microrregión. Estos autores llegaron a esta conclusión a partir de la ubicación de los paneles con arte rupestre, al observar que casi siempre se hallan próximos a asentamientos donde los pobladores realizaban diversas actividades, relacionados con espacios productivos y áreas de explotación de recursos (prácticas de pastoreo, agricultura y caza/recolección) y/o con vías de circulación, como es el caso de CG1.

En este sentido, el motivo de caravana observado en el panel 2, podría permitir la interpretación de que una de las múltiples actividades desarrolladas en CG1 habría estado vinculada a intercambios interregionales, sin que ello implicara necesariamente el paso de caravanas formales. La localización del sitio lo posiciona como un punto clave en las vías de circulación, dado que une a ANS hacia el norte con los salares de la puna central (Salta) y hacia el oeste con los sectores altos de las cuencas del río Copiapó y Quebrada del Salado en la vertiente occidental de los andes (Martel et al. 2017). Esta interpretación del motivo de caravana se enmarca en los procesos de interacciones a distancia que unieron a la microrregión de ANS con las áreas vecinas, tanto los valles mesotermales ubicados al sur y al este como la región del Alto Loa en Chile (Olivera 1992). Para fortalecer esta interpretación, será necesario llevar a cabo prospecciones en el terreno que permitan identificar indicios de tráfico interregional, como vías de circulación, sitios de pernocte y apachetas asociadas a los caminos y materiales de ocupación.

En definitiva, consideramos que es posible que durante el Período Formativo en el sitio CG1 se hayan desarrollado actividades productivas vinculadas a la agricultura y al pastoreo de llamas, así como también prácticas rituales, las cuales no estaban al margen de 
la vida económica de la sociedad que habitó el asentamiento. La proximidad de CG1 a un curso de agua y a pasturas facilitó el asentamiento y el desarrollo de la producción agrícola y del pastoreo en el sector, actividades que, como los principales referentes de la vida económica de este grupo, fueron representadas en el arte rupestre (curso de agua y camélidos). Vinculado a esto, el posible carácter ritual del arte rupestre, como en otras regiones de los Andes, pudo haber sido una forma en que la sociedad recurría a distintos rituales propiciatorios para procurarse la abundancia de agua, recurso de vital importancia para el crecimiento de las pasturas y para el cultivo de plantas domésticas. En definitiva, se deduce un carácter polisémico de los motivos tallados en la roca, como figuraciones directas del paisaje y de la vida económica del grupo humano, así como representaciones de aquello que se buscaba atraer mediante el ritual.

Por último, queremos destacar que durante el análisis de los motivos pudimos observar ciertas particularidades estilísticas que podrían remitir a una forma mediante la cual determinados grupos sociales demarcaban el territorio para asegurar la explotación exclusiva de los recursos naturales (Aschero 2007). En nuestro caso pudimos observar que el motivo de pisada humana del panel 1 muestra la combinación de una técnica de ejecución (el picado plano) con un estilo de representación (la impronta de tres dedos) que no se corresponde con otros motivos de temática similar en la microrregión. Si bien un único motivo no es suficiente para concluir que el arte rupestre de la cuenca del río Mojones presenta características que lo distinguen, es un punto de partida para identificar particularidades que puedan apuntar a la existencia de grupos y/o familias extensas que de esta forma demarcaban su derecho exclusivo a la explotación de los recursos de la cuenca, tal como Aschero et al. (2003) proponen para las quebradas de los ríos Las Pitas y Miriguaca.

A este respecto, proponemos realizar prospecciones a futuro, las cuales nos permitan ampliar la muestra existente hasta el momento $\mathrm{y}$, con ello, generar una imagen más completa de las representaciones realizadas durante el formativo, sus asociaciones regionales y particularidades locales.

Agradecimientos: Este trabajo fue apoyado por el Proyecto Arqueológico de Movilidad Pastoril-Agrícola (PAMPA), financiado por la Fundación Gerda Henkel y el proyecto UBACYT "Cambio climático, impacto ambiental y poblaciones humanas durante el Holoceno Tardío (Puna sur, Argentina): Geoarqueología, Bioarqueología e Interdisciplina". (Área: CAMBIO CLIMÁTICO-PIUBACC, 20620170100009BA). Los autores agradecemos el apoyo logístico de la Municipalidad de Antofagasta de la Sierra y el Instituto Nacional de Antropología y Pensamiento Latinoamericano (INAPL, MCN). Agradecemos también a la Doctora María Pía Falchi, por sus pertinentes observaciones, así como a lxs evaluadorxs, cuyos comentarios permitieron una mejora sustancial del presente trabajo. Por último, nuestro reconocimiento al permanente apoyo de lxs compañerxs del equipo del Proyecto Arqueológico Antofagasta de la Sierra. 


\section{Bibliografía citada}

Ambrosino, G.

2017 Rock Art, Ancestors and Water: The Semiotic Construction of Landscapes in the Central Andes. Tesis de Doctorado, Departamento de Antropología, Universidad de Los Andes.

Aschero, C.

$1999 \mathrm{El}$ arte rupestre del desierto puneño y el Noroeste Argentino. En, Arte rupestre de los Andes de Capricornio, J. Berenguer Rodríguez y F. Gallardo Ibáñez (Edit.), pp. 97-135. Museo Chileno de Arte Precolombino, Santiago de Chile.

2000 Figuras Humanas, Camélidos, y Espacios en la Interacción Circumpuneña. Arte en las rocas. En Arte Rupestre, menhires y piedras de colores en Argentina, M. Podestá y M. de Hoyos (Edit.), pp. 15-44. Sociedad Argentina de Antropología, Buenos Aires.

2006 De cazadores y pastores. El arte rupestre de la modalidad Río Punilla en Antofagasta de la Sierra y la cuestión de la complejidad en la Puna meridional argentina. En Tramas en la Piedra. Producción y usos del arte rupestre, D. Fiore y M. Podestá (Edit.), pp. 103-140. Sociedad Argentina de Antropología, Buenos Aires.

2007 Iconos, Huancas y Complejidad en la Puna Sur Argentina. En Producción y Circulación Prehispánica de Bienes en el sur Andino, A. Nielsen, C. Rivolta, V. Seldes, M. Vázquez y P. Mercolli (Edit.), pp. 135-166. Editorial Brujas, Córdoba.

Aschero, C., Á. Martel, y S. Marcos

2003 El Sitio Curuto 5: Nuevos grabados rupestres en Antofagasta de la Sierra de Catamarca. Edición Especial, Rupestre Digital 5: 17-38.

Aschero, C., Á. Martel y S. López Campeny 2009 El sonido del agua... arte rupestre y actividades productivas. El caso de Antofagasta de la Sierra, Noroeste Argentino. En Crónicas sobre la piedra. Arte rupestre de las Américas, M. Sepúlveda, L. Briones y J. Chacama (Edit.), pp. 257-270. Universidad de Tarapacá, Arica.

Aschero, C. y S. Hocsman

2011 Arqueología de las ocupaciones cazadoras-recolectoras de fines del Holoceno Medio de Antofagasta de la Sierra (Puna Meridional Argentina). Chungara. Revista de Antropología 43: 393-411.

Briones, L., P. Clarkson, A. Díaz y C. Mondaca

1999 Huasquiña, las chacras y los geoglifos del desierto: una aproximación al arte rupestre andino. Diálogo Andino 18: 39-62. 
Castro, V. y V. Varela

1994 Ceremonias de Tierra y Agua. Ritos milenarios Andinos. FONDART -Fundación Andes, Santiago de Chile.

Escola, P., A. Elías, L. Gasparotti y N. Sentinelli

2015 Quebrada del río Miriguaca (Antofagasta de la Sierra, Puna meridional argentina): nuevos resultados de recientes prospecciones. Intersecciones en Antropología 16: 383-396.

Espinosa, G.

1996 Lari y Jamp'atu. Ritual de la lluvia y simbolismo andino en una escena de ate rupestre de Ariquilda 1. Norte de Chile. Chungará. Revista de Antropología 28: 133-157.

Falchi, M. P. y M. Podestá

2015 "Aquí estuvimos, por acá pasamos". Grabados de pisadas y huellas humanas en los desiertos sur andinos. En XIX International Rock Art Conference IFRAO 2015. Symbols in the Landscape: Rock Art and its Context, H. Collado Giraldo y J. J. García Arranz (Edit.), pp. 289312. ARKEOS, Tomar.

Falchi, M. P. y M. Podestá

2019 Escutiformes, plumas y camélidos: arte rupestre de la microrregión Quebrada de Ablomé (Guachipas, Salta). Revista Sociedades de Paisajes Áridos y Semi-Áridos 12 (1): 65-88.

Grant, J., M. Casanova, C. Gentile, N. Flores Abad y D. Olivera

2019 El sitio Corral Grande 1, Antofagasta de la Sierra: Arqueofauna, cerámica y asentamiento en sociedades agropastoriles tempranas de la Puna Sur. En: Actas del XX Congreso Nacional de Arqueología Argentina. Exposición. Universidad de Córdoba, Córdoba.

Joslyn, C.

2013 Representations of Nature in Andean Textiles. Journal of Global Initiatives: Policy, Pedagogy, Perspective 7: 39-54.

Lane K., E. Pomeroy y M. Luján Dávila

2018 Over Rock and Under Stone: Carved Rocks and Subterranean Burials at Kipia, Ancash, AD 1000-1532. Open Archaeology 4: 299-321.

Martel, Á.

2006. Arte rupestre y espacios productivos en el Formativo: Antofagasta de la Sierra (Puna Meridional, Argentina). En Tramas en la Piedra. Producción y usos del arte rupestre, D. Fiore y M. Podestá (Edit.), pp. 157-167. Sociedad Argentina de Antropología, Buenos Aires. 
2010 Arte Rupestre de Pastores y Caravaneros. Estudio Contextual de las Representaciones Rupestres durante el Período Agroalfarero Tardío (900 d.C. - 1480 d.C.) en el Noroeste Argentino. Tesis de Doctorado inédita, Facultad de Filosofía y Letras, Universidad de Buenos Aires.

Martel, Á. y P. Escola

2011 Bloques y Arte Rupestre en la Quebrada de Miriguaca (Depto. Antofagasta de la Sierra, Catamarca, Argentina). Boletín de la SIARB 25: 84-92.

Martel, Á., S. Rodríguez Curletto y E. Del Bel

2012 Arte Rupestre y Espacios de Memoria: Las Representaciones del Sitio Confluencia (Antofagasta de la Sierra, Catamarca, Argentina). Revista de Antropología 25: 121-162.

Martel, Á., D. Zamora y M. Lepori

2017 Tráfico y movilidad caravanera en la Puna Catamarqueña. Una mirada internodal. Estudios Atacameños. Arqueología y Antropología Surandinas 56: 197-223.

Olivera, D.

1992 Tecnología y estrategias de adaptación en el Formativo (agro-alfarero temprano) de la Puna Meridional Argentina. Un caso de estudio: Antofagasta de la Sierra (Pcia. de Catamarca, R.A.). Tesis de Doctorado inédita, Facultad de Ciencias Naturales, Universidad Nacional de La Plata.

1998 Cazadores y pastores tempranos de la Puna Argentina. En Past and Present in Andean Prehistory and Early History, S. Ahlgren, A. Muñoz, S. Sjödin y P. Stenborg (Edit.), pp. 153180. EtnologiskaStudier, Göteborg.

2012. El Formativo en los Andes Sur: La incorporación de la opción productiva. En Interculturalidad y Ciencias: Experiencias desde América Latina, T. de Haro, A. Rocchietti, M. A. Runcio, O. Hernández De Lara, M. V. Fernández (Edit.), pp 15-49. Centro de Investigaciones Precolombinas, Buenos Aires.

Olivera, D. y M. Podestá

1993 Los recursos del arte: arte rupestre y sistemas de asentamiento-subsistencia formativos en la Puna Meridional Argentina. Arqueología 3: 93-141.

Olivera, D., P. Tchilinguirian y L. Grana

2004 Paleoambiente y arqueología en la Puna Meridional Argentina: archivos ambientales, escalas de análisis y registro arqueológico. Relaciones de la SAA 29: 229-247.

Olivera, D., A. Elías, M. Pérez y P. Salminci

2015 Corral Grande 1 y Arroyo Seco: Nuevos Aportes al Formativo de Antofagasta de la Sierra (Provincia de Catamarca, Puna Meridional Argentina). Comechingonia. Revista de Arqueología 19: 37-66. 
Podestá, M., L. Manzi, A. Horsey y M. P. Falchi

1991 Función e interacción a través del análisis temático en el arte rupestre. En El arte rupestre en la arqueología contemporánea, M. Podestá, S. Renard de Coquet y M. I. Hernández Llosas (Edit.), pp.: 40-52. Salón Gráfico Integral, Buenos Aires.

Podestá, M. y M. P. Falchi

2015 Suris, camélidos, felinos y otras huellas. Simbología y contexto arqueológico en el arte rupestre sur andino. En XIX International Rock Art Conference IFRAO 2015. Symbols in the Landscape: Rock Art and its Context, H. Collado Giraldo y J. J. García Arranz (Edit.), pp. 2191-2218. ARKEOS, Tomar.

Quiroga, A.

2017 [1929] Folklore Calchaquí. Fundación de Historia Natural Félix de Azara, Buenos Aires.

Seibold, K.

1992 Textiles and cosmology in Choquechancha. En Andean Cosmologies through the time: Persistence and Emergence, R. Dover, K. Seibold y J. McDowell (Edit.), pp. 166-201. Bloomington: Indiana University Press, Indiana.

Tchilinguirian, P., L. Grana y D. Olivera 2018 Contexto paleoambiental para la ocupación arqueológica en Corral Grande (Antofagasta de la Sierra, Catamarca, Argentina). Chungará. Revista de Antropología 50: 605621. 